VOL. 46 (1992) [317-333]

\title{
GENERALISATION OF AN INEQUALITY OF C.T. CHUANG TO VECTOR MEROMORPHIC FUNCTIONS
}

\author{
INDRAJIT LAHIRI
}

We generalise Chuang's inequality to vector meromorphic functions, which is originally a sort of extension of Nevanlinna's second fundamental theorem.

\section{INTRODUCTION}

In Nevanlinna's theory of meromorphic functions the problem of generalising Nevanlinna's second fundamental theorem, replacing the complex constants by "small meromorphic functions", is of great interest to mathematicians. Nevanlinna himself [5, p.47] first posed this problem and solved it in a very restricted case, for only three small functions. His theorem may be stated as follows:

Theorem A. (see [5, Theorem 2.5, p.47]). If $f(z)$ is a nonconstant meromorphic function in the plane and $a_{1}(z), a_{2}(z), a_{3}(z)$ are distinct meromorphic functions satisfying for $\nu=1,2$, and 3

then

$$
\begin{aligned}
T\left\{r, a_{\nu}(z)\right\} & =o\{T(r, f)\} \quad \text { as } r \rightarrow \infty \\
\{1+o(1)\} T(r, f) & \leqslant \sum_{\nu=1}^{3} \bar{N}\left(r, \frac{1}{f-a_{\nu}(z)}\right)+S(r, f)
\end{aligned}
$$

as $r \rightarrow \infty$, where $S(r, f)=O\{\log T(r, f)+\log r\}$ as $r \rightarrow \infty$ through all values if $f(z)$ has finite order and as $r \rightarrow \infty$ outside a set of finite linear measure otherwise.

Then Dufresnoy [3] and Hiong [6] proceeded further with this problem by solving it with a finite number of polynomials. In 1964 Chuang [1], making an intelligent use of a certain Wronskian, was able to solve the problem with a finite number of 'small meromorphic functions', but for a function having comparatively fewer number of poles. Still now a number of authors $[2,4,10,11]$ are working on this problem and on the Wronskian, first appeared in [1]. We may state Chuang's theorem as follows:

Received 26 September 1991

Copyright Clearance Centre, Inc. Serial-fee code: 0004-9729/92 \$A2.00+0.00. 
THEOREM B. [1] Let $f(z)$ be a nonconstant meromorphic function. Let $\psi_{l}(z)$ $(l=1,2, \ldots, p ; p \geqslant 1)$ be $p$ linearly distinct meromorphic functions satisfying

$$
T\left(r, \psi_{l}\right)=o\{T(r, f)\} \quad \text { as } r \rightarrow \infty, \quad l=1,2, \ldots, p
$$

and their $q$ linearly distinct combinations with constant coefficients be

$$
\phi_{j}(z)=\sum_{k=1}^{p} C_{j k} \psi_{k}(z), \quad j=1,2, \ldots, q ; q \geqslant 2
$$

Then we get the inequality

$$
[q-1-o(1)] T(r, f)<\sum_{j=1}^{q} \bar{N}_{p}\left(r, \frac{1}{f-\phi_{j}}\right)+p \bar{N}(r, f)+S(r, f)
$$

where $S(r, f)=O\{\log T(r, f)+\log r\}$ as $r \rightarrow \infty$ through all values if $f(z)$ is of finite order and outside a set of finite linear measure otherwise, and

$$
\bar{N}_{p}(r, f)=\int_{0}^{r} \frac{\bar{n}_{p}(t, f)-\bar{n}_{p}(o, f)}{t} d t+\bar{n}_{p}(o, f) \log r
$$

$\bar{n}_{p}(t, f)$ is the number of poles of $f(z)$ in $|z| \leqslant t$, poles of order $m$ being counted $m$ times if $m \leqslant p$ and $p$ times if $m>p$.

The book [12] perhaps is the only one to contain elaborate discussions on the Nevanlinna theory of vector valued meromorphic functions, which we shall call vector meromorphic functions. The book [12] contains generalisations of Nevanlinna's first and second fundamental theorems to vector meromorphic functions. Therefore Nevanlinna's above problem on his second fundamental theorem is also open for vector meromorphic functions. In this paper we generalise Chuang's inequality to vector mermorphic functions.

\section{DEFINITIONS AND NOTATIONS}

We denote by $C^{n}$ the usual $n$ dimensional complex Euclidean space with the coordinates $\omega=\left(\omega_{1}, \omega_{2}, \ldots, \omega_{n}\right)$, the Hermitian scalar product $(v, \omega)=v_{1} \bar{\omega}_{1}+v_{2} \bar{\omega}_{2}+$ $\ldots+v_{n} \bar{\omega}_{n}, v, \omega \in C^{n}$ and $\bar{\omega}_{i}$ the complex conjugate of $\omega_{i}$. Also the distance between $v, \omega \in C^{n}$ is defined by $\|v-\omega\|=\sqrt{(v-\omega, v-\omega)}$.

If $\omega_{1}=f_{1}(z), \omega_{2}=f_{2}(z), \ldots, \omega_{n}=f_{n}(z)$ be $n$ ( $\left.\geqslant 1\right)$ meromorphic functions of the complex variable $z$ in the Gaussian plane $C^{1}=C$, then in $C$ a vector valued meromorphic function or, in short, a vector meromorphic function is 
given by $f(z)=\left(f_{1}(z), f_{2}(z), \ldots, f_{n}(z)\right)$. A vector valued function is called entire if all of its components are entire. A vector meromorphic function is called transcendental if at least one of its components is a transcendental meromorphic function. A function which is not transcendental is called rational. The function $f^{(\nu)}(z)=\left(f_{1}^{(\nu)}(z), f_{2}^{(\nu)}(z), \ldots, f_{n}^{(\nu)}(z)\right)$ is called the $\nu$ th derivative $(\nu=1,2, \ldots)$ of $f(z)=\left(f_{1}(z), f_{2}(z), \ldots, f_{n}(z)\right)$, and we agree to write $f^{(0)}(z)=f(z)$. If $f(z)=\left(f_{1}(z), f_{2}(z), \ldots, f_{n}(z)\right)$ and $g(z)=\left(g_{1}(z), g_{2}(z), \ldots, g_{n}(z)\right)$ then we define

(i) the sum and difference of $f(z)$ and $g(z)[12]$ as

$$
f(z) \pm g(z)=\left(f_{1}(z) \pm g_{1}(z), \ldots, f_{n}(z) \pm g_{n}(z)\right),
$$

(ii) the product of $f(z)$ and $g(z)[7]$ as

$$
f(z) g(z)=\left(f_{1}(z) g_{1}(z), \ldots, f_{n}(z) g_{n}(z)\right), \quad \text { and }
$$

(iii) the reciprocal of $f(z)[7]$ as

$$
\frac{1}{f(z)}=\left(\frac{1}{f_{1}(z)}, \frac{1}{f_{2}(z)}, \ldots, \frac{1}{f_{n}(z)}\right) \text {. }
$$

A point $z_{0} \in C$ is called a "pole" or " $\infty$-point" of "multiplicity or order $k_{0}$ " of $f(z)=\left(f_{1}(z), \ldots, f_{n}(z)\right)$ if $z_{0}$ is a pole of maximum order $k_{0}$ of at least one of the component functions $f_{k}(z)$. A point $z_{0} \in C$ is called a "zero" of $f(z)$ of "multiplicity or order $k_{0}$ " if all the component functions $f_{1}(z), f_{2}(z), \ldots, f_{n}(z)$ have zeros at $z_{0}$ with the minimum multiplicity $k_{0}$. A zero of multiplicity $k_{0}$ of the vector meromorphic function $f(z)-a, a \in C^{n}, a \neq \infty$, is called an " $a$ point" of $f(z)$ of "multiplicity $k_{0}$ ".

In the paper we denote by $\widehat{z}$ the $n$ tuple $(z, z, \ldots, z)$, where $z \in C[7]$. We now introduce the following definition which will be needed in the sequel.

DEFINITION 1: Let $f(z)$ be a vector meromorphic function and $\bar{n}_{p}(t, \widehat{O} ; f)$ be the number of zeros of $f(z)$ in $|z| \leqslant t$, zeros of order $m$ being counted $m$ times if $m \leqslant p$ and $p$ times if $m>p$. We now define $\bar{N}_{p}\left[r, \widehat{O}_{;} f\right]$ as

$$
\bar{N}_{p}[r, \widehat{O} ; f]=\int_{0}^{\gamma} \frac{\bar{n}_{p}(t, \widehat{O} ; f)-\bar{n}_{p}(0, \widehat{O} ; f)}{t} d t+\bar{n}_{p}(0, \widehat{O} ; f) \log r .
$$

In this paper by the symbol " $r \rightarrow \infty(O, f)$ " we mean the phrase " $r$ tends to infinity through all values if $f(z)$ is of finite order and outside a set of finite linear measure otherwise". Throughout, we shall assume $f(z), \phi(z), a_{\nu}(z)$ et cetera to be vector meromorphic functions, unless otherwise stated and for example, the component functions of $f(z)$ are $f_{1}(z), f_{2}(z), \ldots, f_{n}(z)$. Further definitions and notations which are not explained here may be seen in [12] from where the relevant portions are also noted in [7]. 


\section{KNOWN RESULTS}

In this section we state some results from [7] and [8] which will be needed in the sequel and which are not available in [12].

LEMmA 1. [7] If $f(z)$ and $g(z)$ are meromorphic, then

(i) $m(r, \widehat{O} ; f) \leqslant m(r, 1 / f)$ and

(ii) $m(r, f g) \leqslant m(r, f)+m(r, g)$.

Lemma 2. [8] If $f(z)$ and $g(z)$ are meromorphic, then

(i) $m(r, f \pm g) \leqslant m(r, f)+m(r, g)+O(1)$,

(ii) $N(r, f \pm g) \leqslant N(r, f)+N(r, g)$,

(iii) $\bar{N}(r, f \pm g) \leqslant \bar{N}(r, f)+\bar{N}(r, g)$,

(iv) $N(r, f g) \leqslant N(r, f)+N(r, g)$,

(v) $\bar{N}(r, f g) \leqslant \bar{N}(r, f)+\bar{N}(r, g)$,

(vi) $T(r, f \pm g) \leqslant T(r, f)+T(r, g)+O(1)$, and

(vii) $T(r, f g) \leqslant T(r, f)+T(r, g)$.

Theorem C. (Generalised Milloux Theorem) [8]. Let $f(z)$ be a nonconstant meromorphic function, $l$ be a positive integer and $\psi(z)=\sum_{\nu=0}^{l} a_{\nu}(z) f^{(\nu)}(z)$, where $T\left\{r, a_{\nu}(z)\right\}=o\{T(r, f)\}$ as $r \rightarrow \infty(O, f), \nu=0,1,2, \ldots, l$. Then as $r \rightarrow \infty$ $(O, f)$

(i) $m(r, \psi / f)=o\{T(r, f)\}$, and

(ii) $T(r, \psi) \leqslant(l+1) T(r, f)+o\{T(r, f)\}$.

\section{Determinants of $n$-TUPLES}

Now we introduce the notion of determinants formed from the elements of $C^{n}$, which will be helpful to define the Wronskians of vector meromorphic functions. To do this, first we need the following definition.

Definition 2: Let $a=\left(a_{1}, a_{2}, \ldots, a_{n}\right)$ and $b=\left(b_{1}, b_{2}, \ldots, b_{n}\right)$ be two elements of $C^{n}$. Then the sum, the difference and the product of $a$ and $b$ are defined respectively by

and

$$
\begin{aligned}
a+b & =\left(a_{1}+b_{1}, a_{2}+b_{2}, \ldots, a_{n}+b_{n}\right), \\
a-b & =\left(a_{1}-b_{1}, a_{2}-b_{2}, \ldots, a_{n}-b_{n}\right), \\
a b & =\left(a_{1} b_{1}, a_{2} b_{2}, \ldots, a_{n} b_{n}\right) .
\end{aligned}
$$

Now we give the main definition of this section. 
Definition 3: Let $a_{i j}=\left(a_{i j}^{1}, a_{i j}^{2}, \ldots, a_{i j}^{n}\right), i, j=1,2, \ldots, m$ be $m^{2}$ elements of $C^{n}$. Then we define the expression

$$
A=\left|\begin{array}{cccc}
a_{11} & a_{12} & \ldots & a_{1 m} \\
a_{21} & a_{22} & \ldots & a_{2 m} \\
\vdots & \vdots & & \vdots \\
a_{m 1} & a_{m 2} & \ldots & a_{m m}
\end{array}\right|
$$

with the supposition that the expansion of $A$ follows the usual rules of expansion of determinants where the addition and multiplication are given by Definition 2, a determinant formed from the elements of $C^{n}$.

Now it is easy to verify that $A=\left(A^{1}, A^{2}, \ldots, A^{n}\right)$, where

$$
A^{k}=\left|\begin{array}{cccc}
a_{11}^{k} & a_{12}^{k} & \ldots & a_{1 m}^{k} \\
a_{21}^{k} & a_{22}^{k} & \ldots & a_{2 m}^{k} \\
\vdots & \vdots & & \vdots \\
a_{m 1}^{k} & a_{m 2}^{k} & \ldots & a_{m m}^{k}
\end{array}\right|, k=1,2, \ldots, n
$$

The following properties of the determinant $A$ are obvious.

I. If two rows or two columns of $A$ are identical, then $A=\widehat{O}$.

II. The interchange of two rows or of two columns of $A$ changes $A$ to $-A$.

III. If the rows of $A$ are changed into columns and the columns of $A$ are changed into rows, then $A$ remains unchanged.

IV. Addition of a complex number multiple of a row (column) of $A$ to another row (column) of $A$ does not change $A$.

V. If any row or column of $A$ is multiplied by a complex number $\alpha$, then $A$ becomes $\alpha A$.

\section{Generalised Chuang's ineQuality}

Now we present two forms of generalisation of the inequality of Chuang (Theorem B) to vector meromorphic functions, each of which coincides with Theorem $B$ for $n=1$, because when $n=1, V(r, a, f) \equiv 0$ for all $a \in C^{n}$ and for every meromorphic function $f(z)[12$, pp.8, 18].

THEOREM 1. Let $f(z)$ be a nonconstant meromorphic function. Let $\psi_{l}(z)$ $(l=1,2, \ldots, p ; p \geqslant 1)$ be $p$ meromorphic functions which are linearly distinct and satisfy

$$
T\left[r, \psi_{l}(z)\right]=o\{T(r, f)\} \quad \text { as } r \rightarrow \infty(O, f), 1=1,2, \ldots, p
$$


322

I. Lahiri

[6]

and their $q$ linearly distinct combinations

$$
\phi_{j}(z)=\sum_{l=1}^{p} c_{j l} \psi_{l}(z), \quad j=1,2, \ldots, q, q \geqslant n+1
$$

with constant coefficients. Then we get the inequality

$$
(q-n) T(r, f) \leqslant \sum_{j=1}^{q}\left\{\bar{N}_{p}\left[r, \widehat{O} ; f-\phi_{j}\right]+V\left[r, \widehat{O} ; f-\phi_{j}\right]\right\}+n p \bar{N}(r, f)+S(r, f)
$$

where $S(r, f)=o\{T(r, f)\}+O(\log r)$ as $r \rightarrow \infty(O, f)$.

ProOF: Let $f(z)=\left(f_{1}(z), f_{2}(z), \ldots, f_{n}(z)\right)$ and $\phi_{j}(z)=\left(\phi_{j 1}(z)\right.$, $\left.\phi_{j 2}(z), \ldots, \phi_{j n}(z)\right), j=1,2, \ldots, q$. Now we set

$$
F(z)=\sum_{j=1}^{q} \frac{1}{f(z)-\phi_{j}(z)}
$$

Let $\mu_{k}(\theta)=\min _{1 \leqslant j_{1}<j_{2} \leqslant q}\left(\left|\phi_{j_{1} k}\left(r e^{i \theta}\right)-\phi_{j_{2} k}\left(r e^{i \theta}\right)\right|, 1\right), O \leqslant \pi \leqslant 2 \pi$ and $k=$ $1,2, \ldots n ;$ and let $\mu(\theta)=\min _{1 \leqslant k \leqslant n} \mu_{k}(\theta)$, where we consider a value of $r$. Also let

$$
E_{j}=\left\{\theta: \theta \in[O, 2 \pi] \quad \text { and } \quad\left|f_{k}\left(r e^{i \theta}\right)-\phi_{j k}\left(r e^{i \theta}\right)\right|<\frac{\mu(\theta)}{2 q \sqrt{n}}, k=1,2, \ldots, n\right\}
$$

Now on $E_{j}$ we get for $h \neq j$

$$
\begin{aligned}
\left|f_{k}\left(r e^{i \theta}\right)-\phi_{h k}\left(r e^{i \theta}\right)\right| & \geqslant\left|\phi_{j k}\left(r e^{i \theta}\right)-\phi_{h k}\left(r e^{i \theta}\right)\right|-\left|f_{k}\left(r e^{i \theta}\right)-\phi_{j k}\left(r e^{i \theta}\right)\right| \\
& \geqslant \mu(\theta)-\frac{\mu(\theta)}{2 q \sqrt{n}} \\
& \geqslant \frac{3}{4} \mu(\theta) .
\end{aligned}
$$

Since

$$
F\left(r e^{i \theta}\right)=\frac{1}{f\left(r e^{i \theta}\right)-\phi_{j}\left(r e^{i \theta}\right)}\left[\widehat{1}+\sum_{h \neq j} \frac{f\left(r e^{i \theta}\right)-\phi_{j}\left(r e^{i \theta}\right)}{f\left(r e^{i \theta}\right)-\phi_{h}\left(r e^{i \theta}\right)}\right]
$$

https://doi.org/10.1017/S000497270001193X Published online by Cambridge University Press 
on $E_{j}$ we have

$$
\begin{aligned}
\left\|F\left(r e^{i \theta}\right)\right\| & \geqslant \frac{1}{\left\|f\left(r e^{i \theta}\right)-\phi_{j}\left(r e^{i \theta}\right)\right\|}\left[1-\sum_{h \neq j}\left\|\frac{f\left(r e^{i \theta}\right)-\phi_{j}\left(r e^{i \theta}\right)}{f\left(r e^{i \theta}\right)-\phi_{h}\left(r e^{i \theta}\right)}\right\|\right] \\
& =\frac{1}{\left\|f\left(r e^{i \theta}\right)-\phi_{j}\left(r e^{i \theta}\right)\right\|}\left[1-\sum_{h \neq j}\left\{\sum_{k=1}^{n}\left|\frac{f_{k}\left(r e^{i \theta}\right)-\phi_{j k}\left(r e^{i \theta}\right)}{f_{k}\left(r e^{i \theta}\right)-\phi_{h k}\left(r e^{i \theta}\right)}\right|^{2}\right\}^{1 / 2}\right] \\
& \geqslant \frac{1}{\left\|f\left(r e^{i \theta}\right)-\phi_{j}\left(r e^{i \theta}\right)\right\|}\left[1-\sum_{h \neq j}\left\{\sum_{k=1}^{n}\left(\frac{\mu(\theta)}{2 q \sqrt{n}} \frac{4}{3 \mu(\theta)}\right)^{2}\right\}^{1 / 2}\right] \\
& =\frac{1}{\left\|f\left(r e^{i \theta}\right)-\phi_{j}\left(r e^{i \theta}\right)\right\|}\left[1-\sum_{h \neq j}\left\{n\left(\frac{2}{3 q \sqrt{n}}\right)^{2}\right\}^{1 / 2}\right] \\
& =\frac{1}{\left\|f\left(r e^{i \theta}\right)-\phi_{j}\left(r e^{i \theta}\right)\right\|}\left[1-\frac{2(q-1)}{3 q}\right] \\
& \geqslant \frac{1}{3} \frac{1}{\left\|f\left(r e^{i \theta}\right)-\phi_{j}\left(r e^{i \theta}\right)\right\|},
\end{aligned}
$$

which gives

$$
\int_{E_{j}} \log ^{+}\left\|F\left(r e^{i \theta}\right)\right\| d \theta \geqslant \int_{E_{j}} \log { }^{+} \frac{1}{\left\|f\left(r e^{i \theta}\right)-\phi_{j}\left(r e^{i \theta}\right)\right\|} d \theta-\log 3
$$

Since $E_{j} \cap E_{s}=\emptyset$ for $j \neq s$ and $\bigcup_{j=1}^{q} E_{j} \subset[0,2 \pi]$, we get

$$
\begin{aligned}
m(r, F) & =\frac{1}{2 \pi} \int_{0}^{2 \pi} \log +\left\|F\left(r e^{i \theta}\right)\right\| d \theta \\
& \geqslant \frac{1}{2 \pi} \sum_{j=1}^{q} \int_{E_{j}} \log ^{+}\left\|F\left(r e^{i \theta}\right)\right\| d \theta \\
& \geqslant \frac{1}{2 \pi} \sum_{j=1}^{q} \int_{E_{j}} \log ^{+} \frac{1}{\left\|f\left(r e^{i \theta}\right)-\phi_{j}\left(r e^{i \theta}\right)\right\|} d \theta-\frac{q}{2 \pi} \log 3 .
\end{aligned}
$$

Let $H_{j}$ be the the complement of $E_{j}$ on $[0,2 \pi]$. Then on $H_{j}$ we get 
$\left|f_{k_{j}}\left(r e^{i \theta}\right)-\phi_{j k_{j}}\left(r e^{i \theta}\right)\right| \geqslant(\mu(\theta)) /(2 q \sqrt{n})$ for some $k_{j}$ in $\{1,2, \ldots, n\}$. So we have

$$
\begin{aligned}
& \frac{1}{2 \pi} \int_{E_{j}} \log ^{+} \frac{1}{\left\|f\left(r e^{i \theta}\right)-\phi_{j}\left(r e^{i \theta}\right)\right\|} d \theta \\
& \quad=m\left[r, \widehat{o} ; f-\phi_{j}\right]-\frac{1}{2 \pi} \int_{H_{j}} \log ^{+} \frac{1}{\left\|f\left(r e^{i \theta}\right)-\phi_{j}\left(r e^{i \theta}\right)\right\|} d \theta \\
& \quad \geqslant m\left[r, \widehat{o} ; f-\phi_{j}\right]-\frac{1}{2 \pi} \int_{H_{j}} \log ^{+} \frac{1}{\left|f_{k_{j}}\left(r e^{i \theta}\right)-\phi_{j k_{j}}\left(r e^{i \theta}\right)\right|} d \theta \\
& \quad \geqslant m\left[r, \widehat{o} ; f-\phi_{j}\right]-\frac{1}{2 \pi} \int_{H_{j}} \log ^{+} \frac{2 q \sqrt{n}}{\mu(\theta)} d \theta \\
& \quad \geqslant m\left[r, \widehat{o}, f-\phi_{j}\right]-\frac{1}{2 \pi} \int_{0}^{2 \pi} \log ^{+} \frac{1}{\mu(\theta)} d \theta-\log (2 q \sqrt{n}) .
\end{aligned}
$$

So from (1) we get

$$
m(r, F) \geqslant \sum_{j=1}^{q} m\left[r, \widehat{O}, f-\phi_{j}\right]-\frac{q}{2 \pi} \int_{0}^{2 \pi} \log ^{+} \frac{1}{\mu(\theta)} d \theta-q \log (2 q \sqrt{n}) .
$$

Again we can easily verify that

$$
\frac{1}{\mu(\theta)} \leqslant 1+\sum_{1 \leqslant j_{1}<j_{2} \leqslant q} \sum_{k=1}^{n} \frac{1}{\left|\phi_{j_{1} k}\left(r e^{i \theta}\right)-\phi_{j_{2} k}\left(r e^{i \theta}\right)\right|}
$$

which gives

$$
\log ^{+} \frac{1}{\mu(\theta)} \leqslant \sum_{1 \leqslant j_{1}<j_{2} \leqslant q} \sum_{k=1}^{n} \log ^{+} \frac{1}{\left|\phi_{j_{1} k}\left(r e^{i \theta}\right)-\phi_{j_{2} k}\left(r e^{i \theta}\right)\right|}+O(1)
$$

that is,

$$
\begin{aligned}
\frac{1}{2 \pi} \int_{0}^{2 \pi} \log ^{+} \frac{1}{\mu(\theta)} d \theta & \leqslant \sum_{1 \leqslant j_{1}<j_{2} \leqslant q} \sum_{k=1}^{n} \frac{1}{2 \pi} \int_{0}^{2 \pi} \log ^{+} \frac{1}{\left|\phi_{j_{1} k}\left(r e^{i \theta}\right)-\phi_{j_{2} k}\left(r e^{i \theta}\right)\right|} d \theta+O(1) \\
& =\sum_{1 \leqslant j_{1}<j_{2} \leqslant q} \sum_{k=1}^{n} m\left(r, \frac{1}{\phi_{j_{1} k}-\phi_{j_{2} k}}\right)+O(1) .
\end{aligned}
$$

Therefore, from (2) we obtain

$$
\begin{aligned}
m(r, F) \geqslant & \sum_{j=1}^{q} m\left[r, \widehat{O} ; f-\phi_{j}\right] \\
& -q \sum_{1 \leqslant j_{1}<j_{2} \leqslant q} \sum_{k=1}^{n} m\left(r, \frac{1}{\phi_{j_{1} k}-\phi_{j_{2} k}}\right)+O(1) .
\end{aligned}
$$


Let $A_{0}=\Delta\left(\psi_{1}, \psi_{2}, \ldots, \psi_{p}\right)$ be the Wronskian determinant of $\psi_{l}(z)$ $(l=1,2, \ldots, p)$, and let $\Delta\left(f, \psi_{1}, \psi_{2}, \ldots, \psi_{p}\right)$ be the Wronskian determinant of $f(z)$, $\psi_{1}(z), \psi_{2}(z), \ldots, \psi_{p}(z)$. Also let

$$
\begin{aligned}
L(f) & =\frac{(-1)^{p}}{A_{0}} \Delta\left(f, \psi_{1}, \psi_{2}, \ldots, \psi_{p}\right) \\
& =f^{(p)}+\frac{A_{1}}{A_{0}} f^{(p-1)}+\ldots+\frac{A_{p-1}}{A_{0}} f^{(1)}+\frac{A_{p}}{A_{0}} f
\end{aligned}
$$

where $A_{l}$ is the cofactor of $f^{(p-1)}$ in $\Delta\left(f, \psi_{1}, \psi_{2}, \ldots, \psi_{p}\right)$.

Now,

$$
\begin{aligned}
F(z) & =\frac{1}{L[f(z)]} \sum_{j=1}^{q} \frac{L[f(z)]}{f(z)-\phi_{j}(z)} \\
& =\frac{1}{L[f(z)]} \sum_{j=1}^{q} \frac{L\left[f(z)-\phi_{j}(z)\right]}{f(z)-\phi_{j}(z)}
\end{aligned}
$$

and so by Lemma 1(ii) and Lemma 2(i) we get

$$
m(r, F) \leqslant m\left[r, \frac{1}{L(f)}\right]+\sum_{j=1}^{q} m\left[r, \frac{L\left(f-\phi_{j}\right)}{f-\phi_{j}}\right]+O(1) .
$$

If $L(f)=\left(L_{1}(f), L_{2}(f), \ldots, L_{n}(f)\right)$, we get in view of [12, Proposition 7.4, p.51] that

$$
\begin{aligned}
m\left[r, \frac{1}{L(f)}\right] & =T\left[r, \frac{1}{L(f)}\right]-N\left[r, \frac{1}{L(f)}\right] \\
& \leqslant \sum_{k=1}^{n} T\left[r, \frac{1}{L_{k}(f)}\right]-N[r, \widehat{O} ; L(f)]
\end{aligned}
$$

because

$$
\begin{aligned}
N\left[r, \frac{1}{L(f)}\right] & \geqslant N[r, \widehat{O} ; L(f)] \\
& =\sum_{k=1}^{n} T\left[r, L_{k}(f)\right]-N[r, \widehat{O} ; L(f)]+O(1),
\end{aligned}
$$

by Nevanlinna's first fundamental theorem $[9$, p.166],

$$
\begin{aligned}
& \leqslant n T[r, L(f)]-N[r, \widehat{O} ; L(f)]+O(1) \\
& =n m[r, L(f)]+n N[r, L(f)]-N[r, \widehat{O} ; L(f)]+O(1)
\end{aligned}
$$


Since by Lemma 1 (ii) $m[r, L(f)] \leqslant m(r, f)+m[r,(L(f)) / f]$, it follows from (6) that

$$
\begin{aligned}
m\left[r, \frac{1}{L(f)}\right] \leqslant & n m(r, f)+n m\left[r, \frac{L(f)}{f}\right]+n N[r, L(f)] \\
& -N[r, \widehat{O} ; L(f)]+O(1) .
\end{aligned}
$$

Combining (5) and (7) we get

$$
\begin{aligned}
m(r, F) \leqslant & n m(r, f)+n m\left[r, \frac{L(f)}{f}\right]+n N[r, L(f)] \\
& -N[r, \widehat{O} ; L(f)]+\sum_{j=1}^{q} m\left[r, \frac{L\left(f-\phi_{j}\right)}{f-\phi_{j}}\right]+O(1) .
\end{aligned}
$$

For a vector meromorphic function $g(z)$ we define the following:

$$
\alpha\left(g, z_{0}\right)=\text { the order of } z_{0} \text { or zero according as } z_{0} \text { is a zero of } g(z) \text { or not, }
$$

and

$$
\beta\left(g, z_{0}\right)=\text { the order of } z_{0} \text { or zero according as } z_{0} \text { is a pole of } g(z) \text { or not. }
$$

If $z_{0}$ is a pole of $L(f)$, from (4) it is clear that $z_{0}$ is a pole of at least one of the functions $f(z), \psi_{l}(z)(l=1,2, \ldots, p)$ and $1 / A_{0}$. So we get

$$
\beta\left[L(f), z_{0}\right] \leqslant \beta\left(f^{(p)}, z_{0}\right)+\sum_{l=1}^{p} \beta\left(\psi_{l}^{(p)}, z_{0}\right)+\beta\left(\frac{1}{A_{0}}, z_{0}\right)
$$

If $z_{0}$ runs over the poles of $L(f)$ within $|z| \leqslant r$, we have

$$
\begin{aligned}
n[r, L(f)] & \leqslant n\left(r, f^{(p)}\right)+\sum_{l=1}^{p} n\left(r, \psi_{l}^{(p)}\right)+n\left(r, \frac{1}{A_{0}}\right) \\
& =n(r, f)+p \bar{n}(r, f)+\sum_{l=1}^{p}\left\{n\left(r, \psi_{l}\right)+p \bar{n}\left(r, \psi_{l}\right)\right\}+n\left(r, \frac{1}{A_{0}}\right)
\end{aligned}
$$

and so

$$
\begin{aligned}
N[r, L(f)] \leqslant & N(r, f)+p \bar{N}(r, f)+\sum_{l=1}^{p}\left\{N\left(r, \psi_{l}\right)+p \bar{N}\left(r, \psi_{l}\right)\right\} \\
& N\left(r, \frac{1}{A_{0}}\right)+O(\log r)
\end{aligned}
$$


Now we shall prove that

$$
\begin{aligned}
& \alpha\left[L(f), z_{0}\right]+\sum_{1 \leqslant j_{1}<j_{2} \leqslant q} \alpha\left[\phi_{j_{1}}-\phi_{j_{2}}, z_{0}\right] \\
& \quad \geqslant \sum_{j-l}^{q}\left\{\alpha\left[f-\phi_{j}, z_{0}\right]-p\right\}^{+}-\sum_{l=1}^{p} \beta\left[\psi_{l}^{(p)}, z_{0}\right]-\beta\left[\frac{1}{A_{0}}, z_{0}\right]
\end{aligned}
$$

where $\left\{\alpha\left[f-\phi_{j}, z_{0}\right]-p\right\}^{+}$is equal to $\alpha\left[f-\phi_{j}, z_{0}\right]-p$ or to zero according as $z_{0}$ is a zero of order greater than $p$ of $f-\phi_{j}$ or not.

Inequality (10) is evident if the first sum on the right hand side of $(10)$ is zero. If this sum is positive, let $\alpha\left[f-\phi_{j_{0}}, z_{0}\right]$ is the maximum of the numbers $\alpha\left[f-\phi_{j}, z_{0}\right]$, $j=1,2, \ldots, q$. Then by means of the relation

$$
f-\phi_{j}=\left(f-\phi_{j 0}\right)-\left(\phi_{j}-\phi_{j 0}\right)
$$

it follows that

$$
\alpha\left[f-\phi_{j}, z_{0}\right] \leqslant \alpha\left[\phi_{j}-\phi_{j_{0}}, z_{0}\right] \quad \text { for } j \neq j_{0},
$$

and hence it remains to verify that

$$
\begin{aligned}
\alpha\left[L(f), z_{0}\right] \geqslant & \left\{\alpha\left[f-\phi_{j_{0}}, z_{0}\right]-p\right\}+ \\
& -\sum_{l=1}^{p} \beta\left(\psi_{l}^{(p)}, z_{0}\right)-\beta\left(\frac{1}{A_{0}}, z_{0}\right)
\end{aligned}
$$

Now (11) follows from the fact that $\alpha\left[L(f), z_{0}\right]=\alpha\left[L\left(f-\phi_{j_{0}}\right), z_{0}\right]$ and if the right hand side of (11) is positive, $m$, say, $z_{0}$ is a zero of order at least equal to $m$ of

$$
\frac{A_{1}}{A_{0}}\left(f-\phi_{j_{0}}\right)^{p-l}, \quad l=1,2, \ldots, p
$$

If $z_{0}$ runs over all the zeros of $L(f)$ in $|z| \leqslant r$, we get from $(10)$ that

$$
\begin{aligned}
& n(r, \widehat{o} ; L(f))+\sum_{1 \leqslant j_{1}<j_{2} \leqslant q} n\left(r, \widehat{O} ; \phi_{j_{1}}-\phi_{j_{2}}\right) \\
& \quad \geqslant \sum_{j=1}^{q} n_{*}\left(r, \widehat{O} ; f-\phi_{j}\right)-\sum_{l=1}^{p}\left\{n\left(r, \psi_{1}\right)+p \bar{n}\left(r, \psi_{1}\right)\right\}-n\left(r, \frac{1}{A_{0}}\right),
\end{aligned}
$$

where $n_{*}\left(r, \widehat{O} ; f-\phi_{j}\right)$ is the number of zeros of order greater than $p$ of $f(z)-\phi_{j}(z)$ situated in the circle $|z| \leqslant r$, each zero is counted the number of times equal to its order minus $\boldsymbol{p}$. 
Then we get

$$
\begin{aligned}
N[r, \widehat{O} ; L(f)]+ & \sum_{1 \leqslant j_{1}<j_{2} \leqslant q} N\left[r, \widehat{O} ; \phi_{j_{1}}-\phi_{j_{2}}\right] \\
\geqslant & \sum_{j=1}^{q} N_{*}\left[r, \widehat{O} ; f-\phi_{j}\right]-\sum_{l=1}^{p}\left\{N\left(r, \psi_{l}\right)+p \bar{N}\left(r, \psi_{l}\right)\right\} \\
& -N\left(r, \frac{1}{A_{0}}\right)+O(\log r)
\end{aligned}
$$

where

$$
\begin{aligned}
N_{*}\left[r, \widehat{O} ; f-\phi_{j}\right]= & \int_{0}^{r} \frac{n_{*}\left(t, \widehat{O} ; f-\phi_{j}\right)-n_{*}\left(0, \widehat{O} ; f-\phi_{j}\right)}{t} d t \\
& +n_{*}\left(0, \widehat{O} ; f-\phi_{j}\right) \log r .
\end{aligned}
$$

Combining (3), (8), (9) and (12) we obtain

$$
\begin{aligned}
\sum_{j=1}^{q} m\left(r, \widehat{O} ; f-\phi_{j}\right) \leqslant n T(r, f)+n p \bar{N}(r, f) & \\
& \quad-\sum_{j=1}^{q} N_{*}\left[r, \widehat{O} ; f-\phi_{j}\right]+S(r, f)
\end{aligned}
$$

where

$$
\begin{aligned}
S(r, f)= & q \sum_{1 \leqslant j_{1}<j_{2} \leqslant q} \sum_{k=1}^{n} m\left(r, \frac{1}{\phi_{j_{1} k}-\phi_{j_{2} k}}\right)+n m\left[r, \frac{L(f)}{f}\right] \\
& +\sum_{j=1}^{q} m\left[r, \frac{L\left(f-\phi_{j}\right)}{f-\phi_{j}}\right]+(1+n) \cdot \sum_{l=1}^{p} N\left(r, \psi_{l}\right) \\
& +p(1+n) \cdot \sum_{l=1}^{p} \bar{N}\left(r, \psi_{l}\right)+(1+n) N\left(r, \frac{1}{A_{0}}\right) \\
& +\sum_{1 \leqslant j_{1}<j_{2} \leqslant q} N\left[r, \widehat{O} ; \phi_{j_{1}}-\phi_{j_{2}}\right]+O(\log r) .
\end{aligned}
$$

Now we investigate the nature of each term in $S(r, f)$.

(i) We have by Nevanlinna's first fundamental theorem [5, p.5, Theorem 1.2], 
[12, p.51, Proposition 7.4] and Lemma 2(vi)

$$
\begin{aligned}
\sum_{k=1}^{n} m\left(r, \frac{1}{\phi_{j_{1} k}-\phi_{j_{2} k}}\right) & \leqslant \sum_{k=1}^{n} T\left(r, \frac{1}{\phi_{j_{1} k}-\phi_{j_{2} k}}\right) \\
& =\sum_{k=1}^{n} T\left(r, \phi_{j_{1} k}-\phi_{j_{2} k}\right)+O(1) \\
& \leqslant n T\left(r, \phi_{j_{1}}-\phi_{j_{2}}\right)+O(1) \\
& \leqslant n T\left(r, \phi_{j_{1}}\right)+n T\left(r, \phi_{j_{2}}\right)+O(1) \\
& =o\{T(r, f)\} \text { as } r \rightarrow \infty(O, f) .
\end{aligned}
$$

So,

$$
q \sum_{1 \leqslant j_{1}<j_{2} \leqslant q} \sum_{k=1}^{n} m\left(r, \frac{1}{\phi_{j_{1} k}-\phi_{j_{2} k}}\right)=o\{T(r, f)\} \text { as } r \rightarrow \infty(O, f) .
$$

(ii) By Theorem $\mathrm{C}$ we get that

$$
n m\left[r, \frac{L(f)}{f}\right]=o\{T(r, f)\} \text { as } r \rightarrow \infty(O, f) .
$$

(iii) Again by Theorem $\mathrm{C}$ we see that

$$
m\left[f \frac{L\left(f-\phi_{j}\right)}{f-\phi_{j}}\right]=o\left\{T\left(r, f-\phi_{j}\right)\right\} \text { as } r \rightarrow \infty(O, f), \quad j=1,2, \ldots, q .
$$

Also, since by Lemma 2 (vi) we have $T\left(r, f-\phi_{j}\right) \leqslant T(r, f)+T\left(r, \phi_{j}\right)$, and by the given hypothesis $T\left(r, \phi_{j}\right)=o\{T(r, f)\}$ as $r \rightarrow \infty(O, f)$, we obtain for $j=1,2, \ldots, q$ that

$$
m\left[r, \frac{L\left(f-\phi_{j}\right)}{f-\phi_{j}}\right]=o\{T(r, f)\} \text { as } r \rightarrow \infty(O, f) .
$$

Therefore,

$$
\sum_{j=1}^{q} m\left[r, \frac{L\left(f-\phi_{j}\right)}{f-\phi_{j}}\right]=o\{T(r, f)\} \text { as } r \rightarrow \infty(O, f)
$$

(iv) Evidently by the given hypothesis we see that $(1+n) \sum_{l=1}^{p} N\left(r, \psi_{l}\right)=$ $o\{T(r, f)\}$ and $p(1+n) \sum_{l=1}^{p} \bar{N}\left(r, \psi_{l}\right)=o\{T(r, f)\}$ as $r \rightarrow \infty(O, f)$.

(v) Let $A_{0}=\left(A_{o 1}, A_{o 2}, \ldots, A_{o n}\right)$. Then we get from the definition of $N\left(r, 1 / A_{\circ}\right)$ that

$$
N\left(r, \frac{1}{A_{\mathrm{o}}}\right) \leqslant \sum_{k=1}^{n} N\left(r, O, A_{o k}\right)
$$


In view of Nevanlinna's first fundamental theorem $[\mathbf{5}, \mathrm{p} .5$, Theorem 1.2], [12, p.51, Proposition 7.4] and Theorem $\mathrm{C}$ we have

$$
\begin{aligned}
(1+n) N\left(r, \frac{1}{A_{o}}\right) & \leqslant(1+n) \sum_{k=1}^{n} N\left(r, O ; A_{o k}\right) \\
& \leqslant(1+n) \sum_{k=1}^{n} T\left(r, A_{o k}\right)+O(1) \\
& \leqslant n(1+n) T\left(r, A_{o}\right)+O(1) \\
& =o\{T(r, f)\} \text { as } r \rightarrow \infty(O, f) .
\end{aligned}
$$

(vi) By the generalised first main theorem [12, p.23, Theorem 3.2], Lemma 2(vi) and by the given hypothesis it follows that

$$
\begin{aligned}
N\left[r, \hat{O}_{;} \phi_{j_{1}}-\phi_{j_{2}}\right] & \leqslant T\left[r, \phi_{j_{1}}-\phi_{j_{2}}\right] \\
& \leqslant T\left(r, \phi_{j_{1}}\right)+T\left(r, \phi_{j_{2}}\right)+O(1) \\
& =o\{T(r, f)\} \quad \text { as } r \rightarrow \infty(O, f) .
\end{aligned}
$$

So, we have

$$
\sum_{1 \leqslant j_{1}<j_{2} \leqslant q} N\left[r, \hat{O}_{;} \phi_{j_{1}}-\phi_{j_{2}}\right]=o\{T(r, f)\} \quad \text { as } r \rightarrow \infty(O, f) .
$$

On account of the above discussions it is now clear from (14) that

$$
S(r, f)=o\{T(r, f)\}+O(\log r) \text { as } r \rightarrow \infty(O, f) .
$$

From the generalised first main theorem [12, p.23, Theorem 3.2] we obtain

$$
m\left[r, \widehat{O} ; f-\phi_{j}\right]=T\left[r, f-\phi_{j}\right]-N\left[r, \widehat{O} ; f-\phi_{j}\right]-V\left[r, \widehat{O} ; f-\phi_{j}\right]+O(1) ;
$$

but since $T\left[r, f-\phi_{j}\right] \geqslant T(r, f)+o\{T(r, f)\}$ as $r \rightarrow \infty(\delta, f)$, we have

$$
\begin{aligned}
\sum_{j=1}^{q} m\left[r, \widehat{O} ; f-\phi_{j}\right] \geqslant & q T(r, f)-\sum_{j=1}^{q}\left\{N\left[r, \widehat{O} ; f-\phi_{j}\right]+V\left[r, \widehat{O} ; f-\phi_{j}\right]\right\} \\
& +o\{T(r, f)\}
\end{aligned}
$$

as $r \rightarrow \infty(O, f)$.

Since $\bar{N}_{p}\left[r, \widehat{O} ; f-\phi_{j}\right]=N\left[r, \widehat{O} ; f-\phi_{j}\right]-N_{*}\left[r, \widehat{O} ; f-\phi_{j}\right]$, the theorem follows by the combination of (13) and (15). This proves the theorem.

For the next theorem we require the following lemma. 
LEMMA 3. If $f(z)$ is a meromorphic function, then for all values of $r$ we get

$$
T\left(r, \frac{1}{f}\right) \leqslant T(r, f)+V\left(r, \widehat{O} ; \frac{1}{f}\right)+O(1) .
$$

Proof: Applying the generalised first main theorem [12, p.23, Theorem 3.2] to $1 /(f(z))$ we get

$$
T\left(r, \frac{1}{f}\right)=m\left(r, \widehat{O} ; \frac{1}{f}\right)+N\left(r, \widehat{O} ; \frac{1}{f}\right)+V\left(r, \widehat{O} ; \frac{1}{f}\right)+O(1)
$$

Since from the definition we get $N(r, \widehat{O} ; 1 / f) \leqslant N(r, f)$ and by Lemma $1(\mathrm{i})$ $m(r, \widehat{O} ; 1 / f) \leqslant m(r, f)$, it follows from above that

$$
T\left(r, \frac{1}{f}\right) \leqslant T(r, f)+V\left(r, \widehat{O} ; \frac{1}{f}\right)+O(1)
$$

This proves the lemma.

Now we prove a second form of generalisation of Theorem B.

THEOREM 2. Let $f(z)$ be a nonconstant meromorphic function. Let $\psi_{l}(z)$ $(l=1,2, \ldots, p ; p \geqslant 1)$ be $p$ meromorphic functions which are linearly distinct and satisfy.

$$
T\left(r, \psi_{l}(z)\right)=o\{T(r, f)\} \quad \text { as } r \rightarrow \infty(O, f), \quad l=1,2, \ldots, p
$$

and their $q$ linearly distinct combinations

$$
\phi_{j}(z)=\sum_{l=1}^{p} c_{j l} \psi_{l}(z), \quad j=1,2, \ldots, q ; q \geqslant 2
$$

with constant coefficients. Then we get the inequality

$$
\begin{gathered}
(q-1) T(r, f) \leqslant \sum_{j=1}^{q}\left\{\bar{N}_{p}\left[r, \widehat{O} ; f-\phi_{j}\right]+V\left[r, \widehat{O} ; f-\phi_{j}\right]\right\}+p \bar{N}(r, f) \\
V\left[r, \widehat{O} ; \frac{1}{L(f)}\right]+S(r, f),
\end{gathered}
$$

where $S(r, f)=o\{T(r, f)\}+O(\log r)$ as $r \rightarrow \infty(O, f)$ and

$$
L(f)=(-1)^{p} \Delta\left(f, \psi_{1}, \psi_{2}, \ldots, \psi_{p}\right) / \Delta\left(\psi_{1}, \psi_{2}, \ldots, \psi_{p}\right)
$$


Proof: By Lemma 3 we get, on noting that $N[r, \widehat{O} ; L(f)] \leqslant N[r, 1 /(L(f))]$,

$$
\begin{aligned}
m\left[r, \frac{1}{L(f)}\right] \leqslant & m[r, L(f)]+N[r, L(f)]-N[r, \widehat{O} ; L(f)] \\
& +V\left[r, \widehat{O} ; \frac{1}{L(f)}\right]+O(1)
\end{aligned}
$$

Since by Lemma $1(i i) m[r, L(f)] \leqslant m(r, f)+m[r,(L(f)) / f]$, it follows from (5) and (16) that

$$
\begin{aligned}
m(r, F) \leqslant & m(r, f)+m\left[r, \frac{L(f)}{f}\right]+N[r, L(f)]-N[r, \widehat{O} ; L(f)] \\
& +\sum_{j=1}^{q} m\left[r, \frac{L\left(r-\phi_{j}\right)}{f-\phi_{j}}\right]+V\left[r, \widehat{O} ; \frac{1}{L(f)}\right]+O(1)
\end{aligned}
$$

In view of (3), (9), (12) and (17) we get

$$
\begin{aligned}
\sum_{j=1}^{q} m\left[r, \widehat{O} ; f-\phi_{j}\right] \leqslant & T(r, f)+p \bar{N}(r, f)+V\left[r, \widehat{O} ; \frac{1}{L(f)}\right] \\
& -\sum_{j=1}^{q} N_{*} \cdot\left[r, \widehat{O} ; f-\phi_{j}\right]+S(r, f),
\end{aligned}
$$

where $S(r, f)$ is given by (14) with $n$ replaced by 1 .

Combining (15) and (18) we get the theorem. This completes the proof of the theorem.

REMARK 1. If, in particular, $f(z)$ and $\psi_{l}(z)(l=1,2, \ldots, p)$ are such that $\lim _{r \rightarrow \infty}(V[r, \widehat{O} ; 1 / L(f)]) /(T[r, 1 / L(f)])=0$ that is, $\delta_{v}(\widehat{O} ; 1 /(L(f)))=1$, then Theorem 2 takes the following form:

For all sufficiently large values of $r$ possibly outside a set of finite linear measure we get

$$
\begin{aligned}
(q-1-\varepsilon) T(r, f) \leqslant & \sum_{j=1}^{q}\left\{\bar{N}_{p}\left[r, \widehat{O} ; f-\phi_{j}\right]+V\left[r, \widehat{O} ; f-\phi_{j}\right]\right\} \\
& +(1+\varepsilon) p \bar{N}(r, f)+S(r, f)
\end{aligned}
$$

where $\varepsilon>0$ if $n>1, \varepsilon=0$ if $n=1$ and $S(r, f)=o\{T(r, f)\}+O(\log r)$ as $r \rightarrow \infty$ $(O, f)$. 


\section{REFERENCES}

[1] Chi tai Chung, 'Une généralisation d'une inégalité de Nevalinna', Scientia Sinica XIII (1964), 887-895.

[2] C. Dai and C.C. Yang, 'On the growth of linear differential polynomials of meromorphic functions', J. Math. Anal. Appl. 150 (1990), 79-84.

[3] J. Dufresnoy, 'Sur les valeurs exceptionnelles des fonctions méromorphes voisines d'une fonction méromorphe donnée', C.R. Acad. Sci. 208 (1939), 255.

[4] G. Frank and G. Weissenborn, 'On the zeros of linear differential polynomials of meromorphic functions', Complex Variables Theory Appl. 12 (1989), 77-81.

[5] W.K. Hayman, Meromorphic functions (The Clarendon Press, 1964).

[6] K.L. Hiong, 'Sur les fonctions méromorphes et les fonctions algébroides', Mémor. Sc. Math. Fase CXXXIX, 30.

[7] I. Lahiri, 'Milloux theorem and deficiency of vector valued meromorphic functions', $J$. Indian Math. Soc. 55 (1990), 235-250.

[8] I. Lahiri, 'Milloux theorem, deficiency and fixpoints of vector valued meromorphic functions', communicated.

[9] R. Nevanlinna, Analytic functions (Springer Verlag, Berlin, Heidelberg, New York, 1970).

[10] N. Steinmentz, 'Eine Verallgemeinerung des zweiten Nevanlinnaschen Hauptsatzes', J. Reine Angrew Math. 368 (1986), 134-141.

[11] N. Steinmentz, 'On the zeros of a certain Wronskian', Bull. London Math. Soc. 20 (1988), 525-531.

[12] H.J.W. Ziegler, Vector valued Nevanlinna theory (Pitman Advanced Publishing Program, 1982).

Department of Mathematics

Jadavpur University

Calcutta 700032

India 